\title{
Molecular characterization of Bifidobacterium longum biovar longum NAL8 plasmids and construction of a novel replicon screening system
}

\author{
Simone Guglielmetti • Matti Karp • Diego Mora • \\ Isabella Tamagnini $\cdot$ Carlo Parini
}

Received: 9 October 2006 / Revised: 8 November 2006 / Accepted: 9 November 2006

(C) Springer-Verlag 2006

\footnotetext{
Electronic supplementary material Supplementary material is available in the online version of this article at http://dx.doi.org/ $10.1007 / \mathrm{s} 00253-006-0755-1$ and is accessible for authorized users only.
}

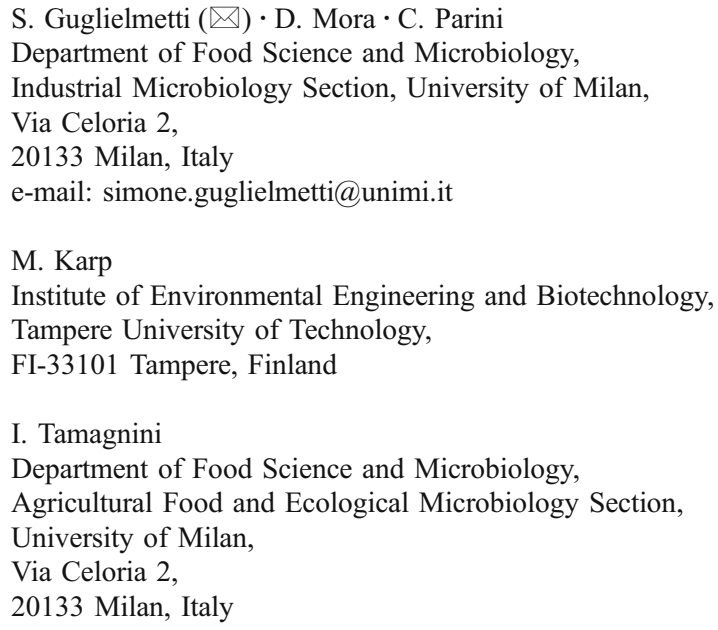

\begin{abstract}
In this study, we performed molecular characterization and sequence analysis of three plasmids from the human intestinal isolate Bifidobacterium longum biovar longum NAL8 and developed a novel vector screening system. Plasmids pNAL8H (10 kb) and pNAL8M (4.9 kb) show close sequence similarity to and the same gene organization as the already characterized $B$. longum plasmids. The $B$. longum plasmid pNAC1 was identified as being most closely related to pNAL8L $(3.5 \mathrm{~kb})$. However, DNA sequence analysis suggested that direct repeat-rich sites could have promoted several recombination events to diversify the two plasmid molecules. We verified the likely rolling circle replication of plasmid pNAL8L and studied the phylogenetic relationship in all the Bifidobacterium plasmids fully sequenced to date based
\end{abstract}

on in silico comparative sequence analysis of their replication proteins and iteron regions. Our transformation experiments confirmed that the ColE1 replication origin from high-copy-number pUC vectors could interfere with the replication apparatus of Bifidobacterium plasmids and give rise to false positive clones. As a result, we developed a system suitable for avoiding possible interference by other functional replication modules on the vector and for screening functional replicons from wildtype plasmids.

\section{Introduction}

Bifidobacteria are becoming commercially and economically significant ingredients in the fermented dairy products and food supplement industries because of their claimed probiotic properties (Stanton et al. 2001).

Studying bacterial plasmids can provide valuable information to help elucidate the ecological mechanisms of microbial adaptability and evolution, and a starting point for preparing instruments for biotechnological purposes.

Only a few Bifidobacterium species have been demonstrated to harbor extrachromosomal DNA molecules, and these species are asteroides, B. breve, B. globosum, $B$. indicum, B. longum, and B. pseudocatenulatum (Schürch 2002; Iwata and Morishita 1989; Sgorbati et al. 1982). Among the above species, plasmids have been most frequently identified in B. longum, a common human intestinal inhabitant that shows a plasmid-bearing frequency of about $68 \%$ of the strains (Sgorbati et al. 1982). Moreover, among the 17 Bifidobacterium plasmid sequences deposited in the GenBank, 12 belong to B. longum.

This study reports molecular characterization and computational sequence analysis of three plasmids in the human 
intestinal isolate B. longum biovar longum NAL8 (Canzi et al. 2005) with an in silico comparison of all the Bifidobacterium plasmid sequences available to date.

As the genetic transformation of bifidobacteria is difficult (Corneau et al. 2004) and the expression of heterologous genes are often unsuccessful (Rossi et al. 1996), few molecular instruments are today available for study and genetic modification of bifidobacteria (Park et al. 1999; Rossi et al. 1996). When we developed various cloning vectors built in the Escherichiacoli strain XL1 based on the replication system ColE1 of pUC18 or pUC19 vectors, we obtained false positive clones not containing the expected recombinant molecule. Other authors (Schürch 2002; Corneau et al. 2004) have reported similar results. Therefore, for our study, we developed and used a novel system suitable to circumvent this problem.

\section{Materials and methods}

Plasmids, bacterial strains, and media

Table 1 lists the bacterial strains and plasmids used in this study. Bifidobacterium strains were grown in MRS (Difco, Detroit, MI, USA) supplemented with $0.05 \%$ L-cysteine hydrochloride (cMRS) anaerobically at $37^{\circ} \mathrm{C}$. E. coli was cultivated in synthetic-oil-based medium at $37^{\circ} \mathrm{C}$. Antibiotics (supplied by Sigma, St. Louis, MO) were employed in the following concentrations: $E$. coli, 200- $\mu$ g erythromy$\operatorname{cin}(\mathrm{Em}) \mathrm{ml}^{-1}, 10-\mu \mathrm{g}$ kanamycin $\mathrm{ml}^{-1}, 6-\mu \mathrm{g}$ chloramphenicol $(\mathrm{Cm}) \mathrm{ml}^{-1}$; Bifidobacterium, 5- $\mu \mathrm{g} \mathrm{Cm} \mathrm{ml} \mathrm{Cl}^{-1}$, and 5- $\mu \mathrm{g}$ $\mathrm{Em} \mathrm{ml}^{-1}$.

Plasmid DNA isolation and analysis

Plasmid DNA isolation from $30 \mathrm{ml}$ of Bifidobacterium broth culture was performed by extracting it with an equal volume of 5-M $\mathrm{LiCl}$ and by precipitating it with polyethylene glycol (Sambrook and Russell 2001). Plasmids were purified by centrifugation to equilibrium in a $\mathrm{CsCl}-\mathrm{EtBr}$ density gradient. Subsequently, the plasmids from the strain NAL8 were separated by electrophoresis and excision from the agarose gel.

For the analysis of circular covalently closed (CCC) and open circle plasmid forms, second-dimension electrophoresis was performed according to Hintermann et al. (1981).

\section{Characterization and sequencing of B. longum NAL8 plasmids}

A complete DNA sequence was determined for plasmids pNAL8M and pNAL8L, whereas for plasmid pNAL8H, a restriction map was prepared by digesting it with restriction enzymes AvaI, EcoRI, EcoRV, KpnI, HincII, HindIII, and PstI (Sigma). Moreover, in pNAL8H, regions A and B were PCR-amplified with primers designed on the DNA sequence of plasmid pNAC3 (NC_004768). PCR reactions were performed with Taq DNA polymerase (Fermentas, Vilnius, Lithuania). Sequences were determined using an ABI Prism BigDye ${ }^{\mathrm{TM}}$ terminator technology in an $\mathrm{ABI}$ Prism $^{\text {TM }} 310$ DNA sequencer (Applied Biosystems, Foster City, CA, USA).

\section{Sequence analysis}

To predict the location of putative open-reading frames (ORFs), an ORF-finder (http://www.ncbi.nlm.nih.gov/gorf/ gorf.html) software was set at default for the bacterial code. The basic local alignment search tool (BLAST) programs (http://www.ncbi.nlm.nih.gov/blast/) were used to conduct similarity searches against the GenBank and EMBL sequence databases. The gene or protein sequence alignments and neighbor-joining phylogenetic analysis were run using ClustalW and Treecon v1.3b software, respectively.
Table 1 Bacterial strains and plasmids used in the study

\begin{tabular}{|c|c|c|}
\hline & Pertinent characteristics & Source \\
\hline \multicolumn{3}{|l|}{ Bacterial strain } \\
\hline B. longum NAL8 & & Canzi et al. (2005) \\
\hline B. animalis $\mathrm{ScMa} 7$ & Isolated from commercial fermented milk & This study \\
\hline B. longum NCC2705 & & Nestlé Culture Collection \\
\hline E. coli $\mathrm{VE} 7108$ & Gram host of $\mathrm{pG}^{+}$host, pGHori and pGOSBif33 & Maguin et al. (1996) \\
\hline \multicolumn{3}{|l|}{ Plasmids } \\
\hline PNAL8H & $10 \mathrm{~kb}$; natural plasmid from $B$. longum NAL8 & This study \\
\hline PNAL8M & 4920 bp; natural plasmid from B.longum NAL8 & This study \\
\hline PNAL8L & $3489 \mathrm{bp}$; natural plasmid from $B$. longum NAL8 & This study \\
\hline $\mathrm{pG}^{+}$host & $\mathrm{Em}^{\mathrm{r}}$, thermosensitive replicon & Maguin et al. (1996) \\
\hline PGHori & 3202 bp; $\mathrm{pG}^{+}$host without rep $A$ gene & This study \\
\hline PGOSBif33 & $6691 \mathrm{bp} ;$ E. coli-Bifidobacterium shuttle vector & This study \\
\hline
\end{tabular}


Plasmid and vector maps were plotted using the Vector NTI 6 (InforMax, Oxford, UK) program.

Detection of single-stranded plasmid DNA

DNA was isolated from B. longum NAL8, treated with nuclease S1 (Roche Diagnostic), separated by agarose gel electrophoresis, and transferred onto a hybond-N nylon membrane (Amersham Biosciences, Milan, Italy; Sambrook and Russell 2001). Single-stranded DNA was identified by using as probe a DNA fragment of 394 bp obtained by PCR from the plasmid pNAL8L (primers employed were p3.5f, 5'-CGGACGGTACTTAGTACAA-3' and SGD3.5r, 5'CCGCAACGTCAACCAGAT-3'). After amplification, the DNA fragment obtained was DIG-dUTP-labeled by random priming with a labeling kit (Roche Diagnostic, Rotkreuz, Switzerland) and used as probe in the hybridization experiment. Filter hybridization was performed according to the supplier's instructions with pre-hybridization and hybridization steps in $50 \%(\mathrm{wt} / \mathrm{vol})$ formamide at $42^{\circ} \mathrm{C}$ and stringency washes in $0.1 \times \mathrm{SSC}, 0.1 \% \mathrm{SDS}$ at $65^{\circ} \mathrm{C}$.

Vector constructions

Unless otherwise stated, ligation reactions were performed in $10 \mu \mathrm{l}$ with $2.5 \mathrm{U}$ of T4 ligase (Fermentas) at $22^{\circ} \mathrm{C}$ for $60 \mathrm{~min}$. E. coli transformations were performed by heat shock of inoue-competent cells (Sambrook and Russell 2001).

To construct the plasmid pGHori, we designed two divergent primers near the extremities of the replication gene of vector $\mathrm{pG}^{+}$host9 (Maguin et al. 1996), pGhf (5' TATCAGTGTGGAGCTCGAGCAAGTT-3'), and pGhr (5' AGCCATAGATGAGCTCAAACTCTCT- $3^{\prime}$ ), which contained the restriction site $\mathrm{SacI}$ (underlined; Fig. 1). The thermosensitive vector $\mathrm{pG}^{+}$host 9 that bears the ribosomal RNA adenine $N$-6-methyltransferase gene for erythromycin resistance was maintained in the E. coli strain VE7108, which has a non-thermosensitive copy of the plasmid replication gene rep $A$ on its chromosome. The PCR product we obtained via amplification with primers pGhf and pGhr, after restriction with $\mathrm{SacI}$, was self-ligated (5 U of Fermentas T4 ligase in a final volume of $50 \mu 1$ ) and cloned back into E. coli VE7108. To obtain vector pGOSBif33, we linearized plasmid pNAL8L with $C l a \mathrm{I}$ and cloned it in the same restriction site of vector pGHori (Fig. 1).

\section{Electroporation of bifidobacteria}

Electrocompetent Bifidobacterium cells were prepared as follows. Fresh cMRS broth $(250 \mathrm{ml})$ supplemented with $0.2 \%$ glucose was inoculated with $10 \mathrm{ml}$ of an overnight culture of the Bifidobacterium strain and incubated at $37^{\circ} \mathrm{C}$ for $1-5 \mathrm{~h}$ until an optical density (at $600 \mathrm{~nm}$ ) of $0.2-0.4$ was reached. The cells were chilled on ice and concentrated in further washing steps of 250,50 , and $10 \mathrm{ml}$ (washing buffer: $1 \mathrm{mmol}^{-1}$ ammonium citrate, $0.5 \%$ sucrose, $\mathrm{pH} 6$ ). After washing, the pellet was resuspended in $1.25 \mathrm{ml}$ of the same buffer. Cell suspensions were stored at $-4^{\circ} \mathrm{C}$ for $3 \mathrm{~h}$ and then maintained at $-80^{\circ} \mathrm{C}$ until used for electroporation $(12.5 \mathrm{kV} / \mathrm{cm}, 2-\mathrm{mm}$ cuvettes, time constants obtained between 3.9 and $4.2 \mathrm{~ms}$ ).

\section{Accession numbers}

The EMBL accession numbers of the DNA sequences determined in this study are as follows: region A of plasmid pNAL8H, AM183142; region B of plasmid pNAL8H, AM183143; complete sequence of plasmid pNAL8M, AM183144; complete sequence of plasmid pNAL8L, AM183145.

\section{Results}

Plasmids in B. longum NAL8

The two-dimensional gel electrophoresis revealed three different CCC DNA molecules in a nucleic acid extract of B. longum NAL8 after ultracentrifugation in a $\mathrm{CsCl}-\mathrm{EtBr}$ density gradient (Supplementary Material S1). The supercoiled DNA ladder suggested the molecular weights of the three molecules labeled pNAL8H, pNAL8M, and pNAL8L to be $10,4.9$, and $3.5 \mathrm{~kb}$, respectively.

Molecular characterization of pNAL8H

The restriction map of the plasmid pNAL8H showed a strong similarity with the already characterized $B$. longum plasmid pNAC3 (NC_004768; Corneau et al. 2004), with differences located only inside two small regions labeled A and B that were sequenced. The sequence analysis revealed that only two point mutations differentiate region $\mathrm{A}(675 \mathrm{bp})$ in pNAL8H from the corresponding region in pNAC3. Region $\mathrm{B}(1,381 \mathrm{bp})$ holds the nucleotides just upstream of the putative plasmid rep gene containing direct repeat sequences called iterons that constitute the putative plasmid replication origin. The putative replication origin of pNAC3 consists of eight direct repeats of 11 nucleotides with the sequence $5^{\prime}$ GGTGGCTAAAG- $3^{\prime}$. In pNAL8H, this motif is present nine times because an insertion of 65 bp observed in region $B$ includes an extra iteron sequence.

Nucleotide sequence analysis of plasmid pNAL8M

Plasmid pNAL8M was fully sequenced, consisting of 4,910 bp with a mean $\mathrm{G}+\mathrm{C}$ content of $61.9 \mathrm{~mol} \%$. A 
Fig. 1 Strategy adopted to construct E. coli VE7108-Bifidobacterium shuttle plasmid pGOSBif33

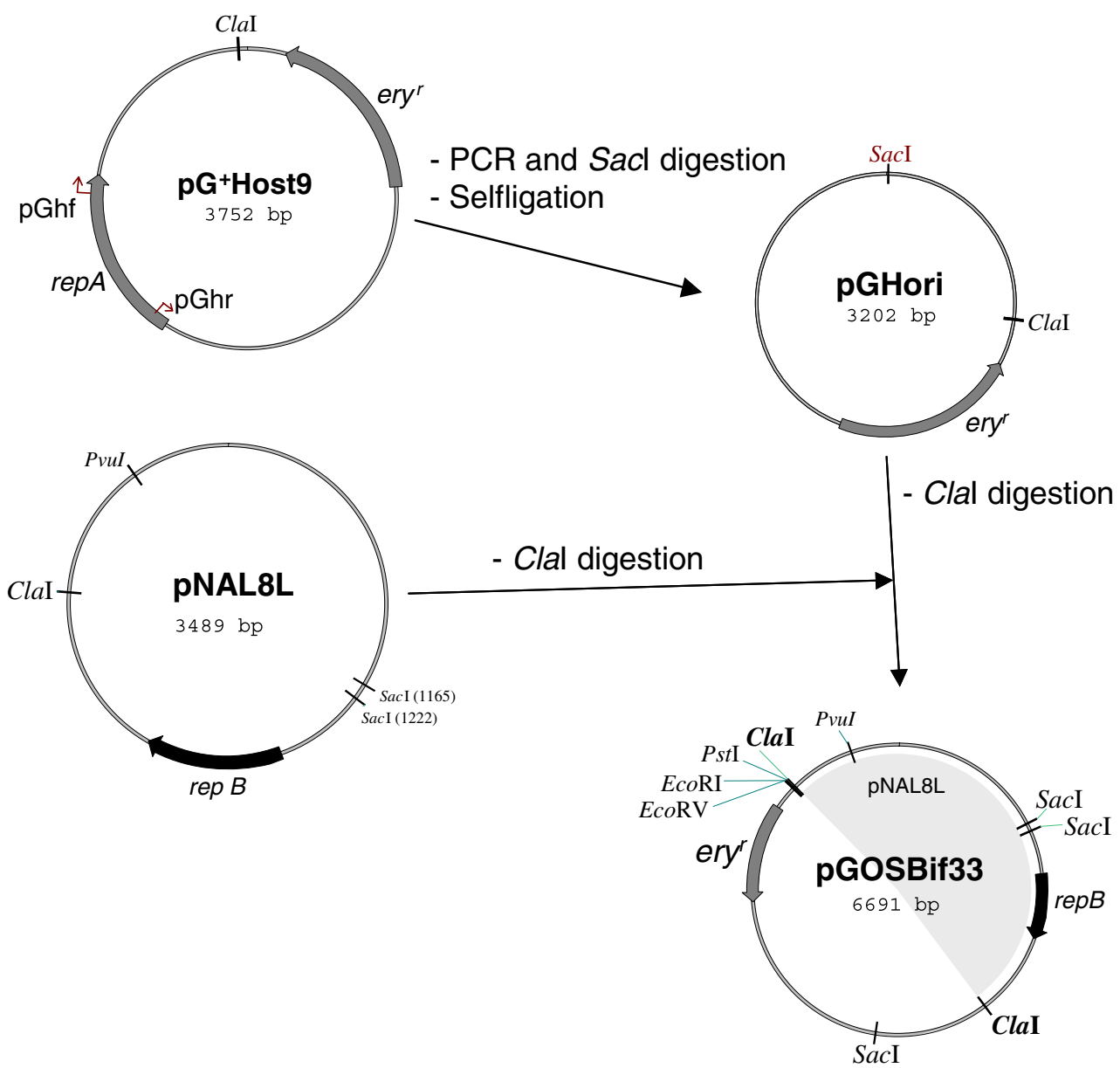

database search revealed that pNAL8M shares $97 \%$ nucleotide identity with pKJ50 (NC_004978), a B. longum plasmid already characterized (Park et al. 1999). Moreover, 4,405 bp of pNAL8M are present with $99 \%$ nucleotide identity in plasmid pDOJH10L. As stated by Lee and O'Sullivan (2006), plasmid pDOJH10L seems to result from the fusion of three different plasmid molecules (Supplementary Material S2). Interestingly, the junctions between the DNA regions of potentially different origin that constitute pDOJH10L are characterized by the $5^{\prime}-\mathrm{CCGG}-3^{\prime}$ motif that may represent the target sequence of recombination events (Supplementary Material S2).

Characterization and sequence analysis of plasmid pNAL8L

The hybridization experiments after S1 nuclease treatment of plasmid DNA revealed that pNAL8L probably replicates through a rolling circle mechanism since we detected the ssDNA of pNAL8L (Supplementary Material S3).

The DNA sequencing of both strands of the plasmid pNAL8L resulted in a single contiguous sequence of 3,489 bp (mean $\mathrm{G}+\mathrm{C}$ content of $59.0 \mathrm{~mol} \%$ ). Five ORFs identified on the molecule showed significant similarity with putative proteins already deposited in the databanks (Fig. 2).

Our DNA sequence analysis showed that pNAC1 is the plasmid molecule most closely related to pNAL8L. However, the new and composite organization of pNAL8L suggests that several recombination events occurred to diversify the two plasmid molecules (Fig. 2). Specifically, the pNAL8L region including orfIII occurs in an orientation opposite to that of the homologous region in pNAC1. This region is delimitated by two loci, $\mathrm{DR}^{\mathrm{II}}$ and $\mathrm{DR}^{\mathrm{III}}$, constituted by direct repeats. $\mathrm{DR}^{\mathrm{III}}$ comprises a 22-bp sequence 5'-ACTTAGTACAAAAGGGGAACGA-3' (sequence $\mathrm{Rp}$ ) repeated 7 times, whereas the corresponding region in $\mathrm{pNAC} 1$ contains the Rp sequence only once. On the other side of the region containing orfIII, $\mathrm{DR}^{\mathrm{II}}$ comprises the sequence 5'-ATGGGCATCTCCATGTG GATGTCC-3', repeated almost perfectly three times. The same 24-bp motif occurs in the corresponding region of pNAC1, repeated also three times. As suggested for other plasmids (Hofreuter and Haas 2002), such sequence repeats may act as hot spots for recombination and site-specific integration. This assumption is supported by the observation that the region of pNAL8L containing orf $V$, closely similar to a chromosomal region in B. longum NCC2705, is 
Fig. 2 Functional map of plasmid pNAL8L and bi-dimensional representation of pNAL8L regions with significant similarity with pNAC1; identity value reported for each pNAL8L fragment in nucleotide ratio and percentage; the region labeled $\mathrm{CR}$ and common to seven other Bifidobacterium plasmids is indicated. orf $V$ codes for a presumptive peptide that is $62 \%$ identical with the hypothetical protein BL1470 found on the chromosome of Bifidobacterium longum NCC2705 (AAN25265). The putative product of orfIV showed similarity $(83 \%$ identity) to the subunit $\mathrm{K} /$ omega (COG1758) of the DNA-directed RNA polymerase of Bifidobacterium longum DJO10A

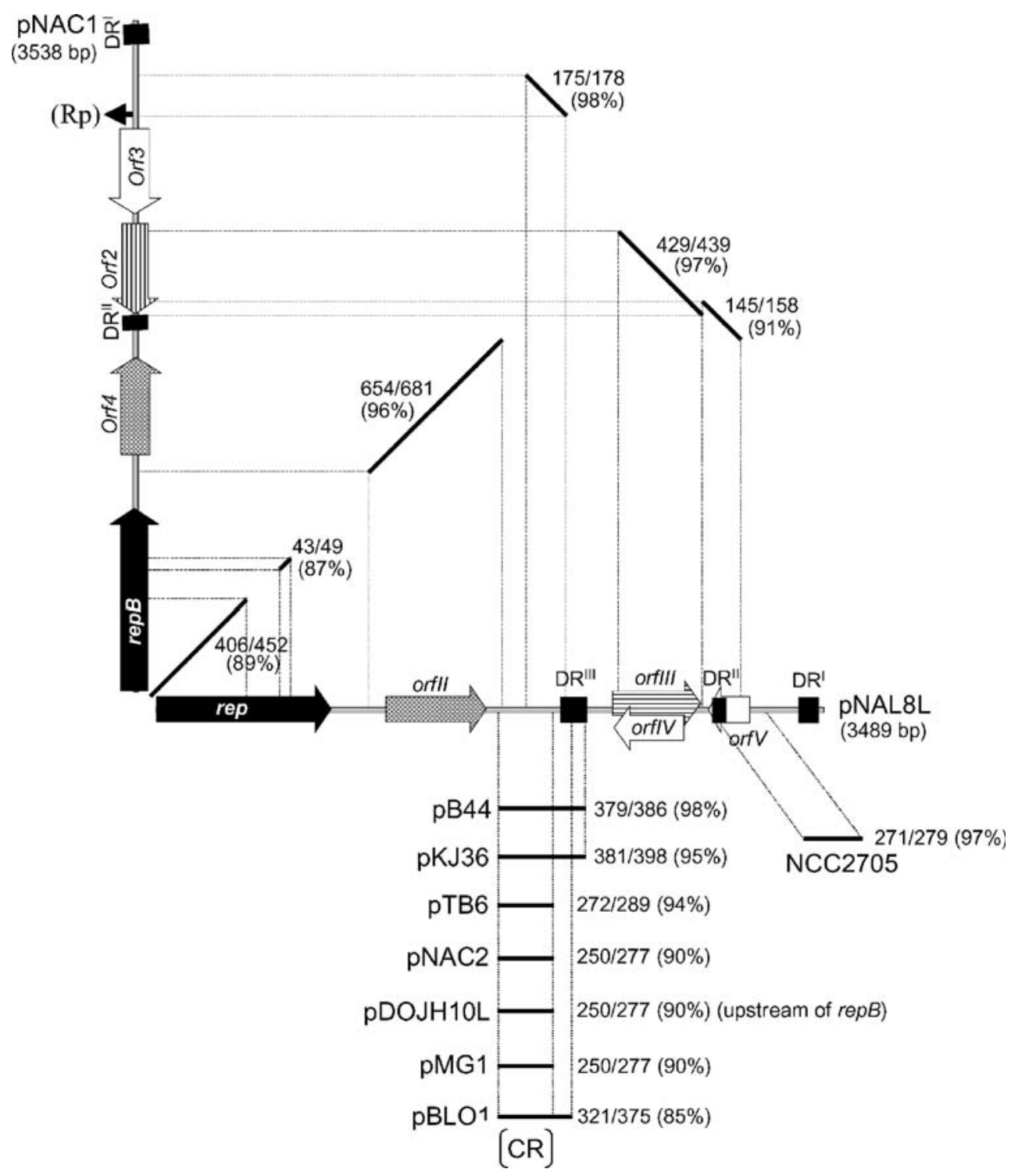

located between two direct repeat-rich sites, $\mathrm{DR}^{\mathrm{I}}$ and $\mathrm{DR}^{\mathrm{II}}$ (Fig. 2).

Furthermore, a region of about $250 \mathrm{bp}$, upstream of the DRIII locus of pNAL8L, was strongly conserved among the 7 plasmids constituting the Rep cluster I (Supplementary Material S4; Fig. 3). This 250-bp DNA fragment contains a 36-bp AT-rich region at its $3^{\prime}$ terminus that may well be the site where DNA strands melt when the plasmids of Rep cluster I start replicating.

Phylogenetic relationship between Bifidobacterium plasmid Rep proteins

With a neighbor-joining dendrogram, Rep proteins from Bifidobacterium plasmids can be clustered into five main groups (Supplementary Material S4 and Fig. 3). B. longum Reps occur in three of these clusters (named I, II, and III), whereas only the replication proteins of two $B$. breve and one B. pseudocatenulatum plasmids constitute groups IV and V, respectively (Supplementary Material S4). This classification is supported by the homology between Reps belonging to a single group and specific Pfam families of initiator protein conserved domains (Supplementary Material S4).

The neighbor-joining dendrogram built, considering Bifidobacterium plasmid Reps and 54 other replication proteins most closely related in the BLAST-p analysis, revealed that each of the five typologies of Bifidobacterium Rep proteins has similarities with clusters of replication proteins from various other hosts (Fig. 3).

Iterons in pNAL8L and other Bifidobacterium plasmids

The iteron region $\mathrm{DR}^{\mathrm{I}}$ of pNAL8L, located just upstream of the rep gene (Fig. 2), shows the typical organization of iteron-containing replication origins (Supplementary Mate- 


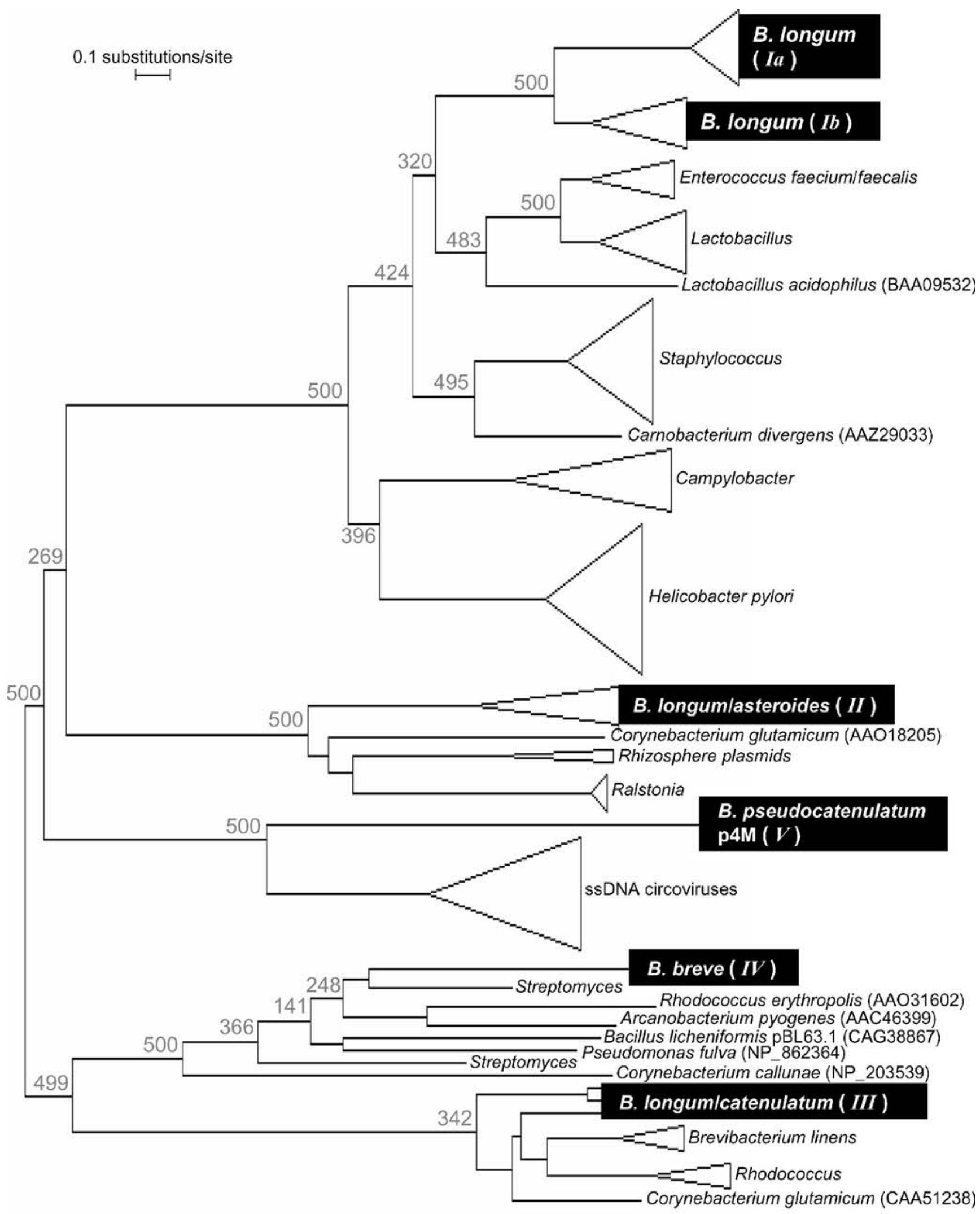


Fig. 3 Neighbor-joining dendrogram of Rep proteins generated from ClustalW alignment of 54 Rep proteins most closely related to 22 Bifidobacterium plasmid Reps in Blast-p search; Bifidobacterium plasmid clusters shown in white letters on black field; bootstrap values of the main internodes (500 replicates) are shown; database accession numbers shown in parentheses. The accession numbers of the replication protein sequences not shown on the dendrogram are as follows: Enterococcus faecium/faecalis, AAL05545, AAL39167, AAM44830, NP 863270; Lactobacillus, AAL54831, AAZ13604, BAA08501, BAA87064, NP_862269, NP_862285; Staphylococcus, AAC63222, AAD02381, AĀD02388, AAD02408A, AAN71829, AAK73551, BAE05993, NP 863200, NP 395563, NP 976281, YP_187551; Campylobacter, AAG40755, BAD95842, CAA57593, CAA 57596 , NP 862911, YP 247575; Helicobacter pylori, AAD11553, AAD11557, AAD11558, AAD11559, AAD11561, AAD11562, ABA26021, ABB51115, NP_044345, NP_858075, NP 862334, NP 862357, YP 025604; plasmids from rhizosphere, CAC79146, CAC82776; Ralstonia, AAZ64686, CAD17152, ZP 00166294, ZP 00596502; circoviruses, AAC69861, AAG30561, AAG30564, AAS 93271 , AAT00476, АAT00477, АAT00478, AAX10150, AAY81718, NP_573442; Brevibacterium linens, CAA72653, NP_065271, ZP_00377816; Rhodococcus, BAE06128, NP_858932

rial S5). It contains three fully identical $22 \mathrm{bp}$ directly repeated sequences followed immediately by an almost perfect, inverted repeated sequence comprising the first $11 \mathrm{bp}$ of the iteron. Just upstream of the direct repeated sequences is an AT-rich region, flanked by smaller GC-rich sequences, where DNA strands may melt at the onset of plasmid replication. Furthermore, plasmid pNAL8L con- tains two other regions with a typical organization of the iteron-containing origins, $\mathrm{DR}^{\mathrm{II}}$ and $\mathrm{DR}^{\mathrm{III}}$, but located far from the replication gene (Fig. 2 and Supplementary Material S5).

When we aligned the iteron regions of the Bifidobacterium plasmids to construct a neighbor-joining dendrogram (Fig. 4), we found significant similarity between $\mathrm{DR}^{\mathrm{II}}$ and iterons from the plasmids of Rep cluster III, whereas $\mathrm{DR}^{\mathrm{III}}$ was significantly similar to iterons from the plasmids of Rep group Ia (Supplementary Material S4; Fig. 3). Globally, the dendrogram in Fig. 4 shows a clear correspondence between the iteron sequence typologies found in Bifidobacterium plasmids and the Rep clusters (Supplementary Material S4). As expected, each Rep protein appears to be associated with its specific iteron region, suggesting that a rep gene and its cognate iteron region should be considered a unique module in the mosaic structure of Bifidobacterium plasmids.

Transformation experiments

We used pGHori vector to prepare an E. coli VE7108Bifidobacterium shuttle plasmid, labeled pGOSBif33, based on pNAL8L. We found no clones when we transformed $B$. longum NAL8L with pGOSBif33, a finding that suggested a possible incompatibility with plasmid pNAL8L, which is naturally present in NAL8. On the contrary, when we (a)

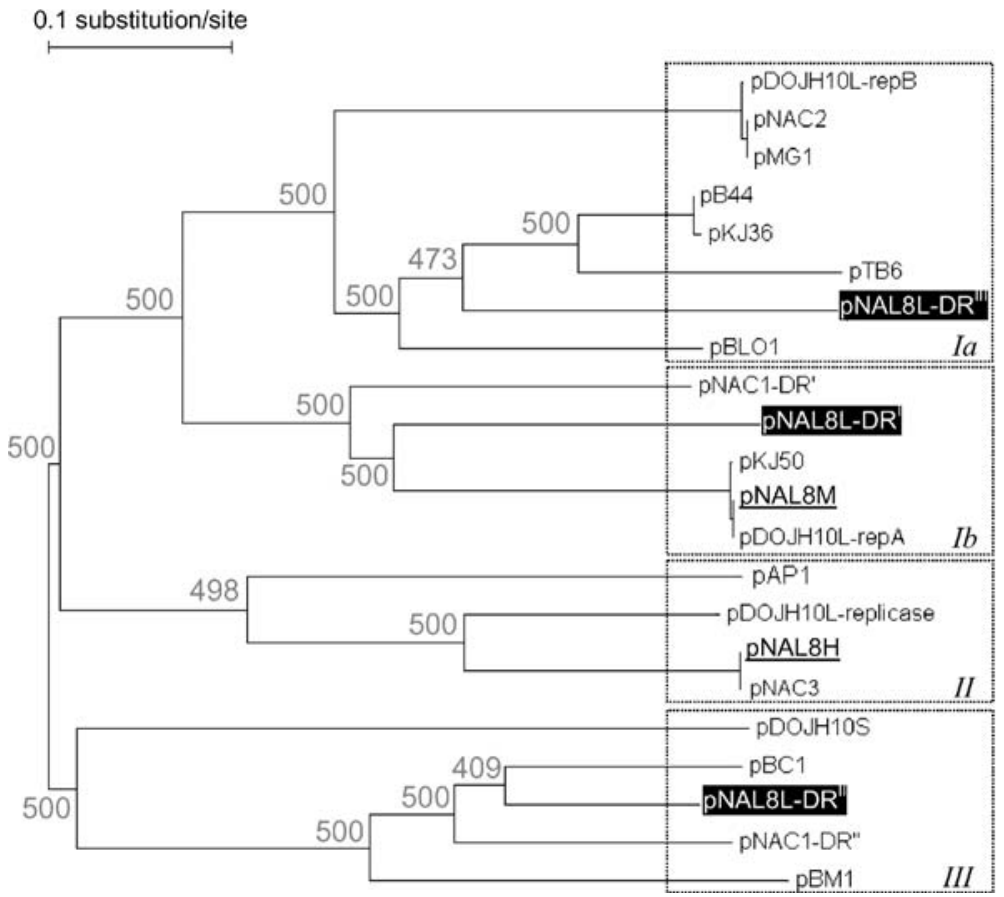

(b)

\begin{tabular}{|c|c|}
\hline pDOJH10L-repB & ACCGGGGACAAAAAGGGAGCGA \\
\hline pMG1 & ACCGGGGACAAAAAGGGAGCGA \\
\hline pNAC2 & ACCGGGGACAAAAAGGGAGCGA \\
\hline $\mathrm{pB} 44$ & ACTTAGTACAAAAGGGGAGCGA \\
\hline pKJ36 & ACTTAGTACAAAAGGGGAGCGA \\
\hline PNAL8L-DR ${ }^{I I I}$ & ACTTAGTACAAAAGGGGARCGA \\
\hline pTB6 & ACCTACACCAAAAGGGGAGCGA \\
\hline pBLO1 & ATTGAGTACAAAATAGGAGCGA \\
\hline consensus & ACYKRgkaCAAAArGGGAgCGA \\
\hline pDOJH10L-repA & ATTAAAGGGGAGATTTTGGGA -..- \\
\hline pNAL8L-DR ${ }^{I}$ & - AAAGGGGACTTTTTGGGAGACC \\
\hline pNAC1 $-\mathrm{DR}^{1}$ & - - AAAAGGGAGATTAAGGACAC-- \\
\hline pKJ50 & - CAAAAAGGGAGCGAACCGGGGA - - \\
\hline pNAL8M & - CAAAAAGGGAGCGAACCGGGGA - - \\
\hline consensus & $\cdots$ AAARGGGAgmKWWYSGg $r \cdots$ \\
\hline pANC3 & -GGTGGCTAAAG \\
\hline pNAL $8 \mathrm{H}$ & -GGTGGCTAAAG \\
\hline pDOJH10L-replicase & YGGTACCTAAAGCACCTCAAA- - - \\
\hline pAP1 & - - TATTTAAAGCCCCTCRRAAYA \\
\hline consensus & -- TRBYTAAAG $\ldots \ldots$ \\
\hline pDOJH10S & $\ldots$ CACGCCCAACC $\ldots$ \\
\hline $\mathrm{pBC} 1$ & ATRGACRTVTCCATGTGGRTRTCC \\
\hline PNAL8L-DR ${ }^{\text {II }}$ & ATGGRCRTSTCCATGTGGRTGTCC \\
\hline pNAC1-DR ${ }^{11}$ & ATGGGCATCTCCATGTGGRTGTCC \\
\hline pMB1 & ATGGAGATATCCATGTGGGTGTCC \\
\hline conse & catntCCAtgt \\
\hline
\end{tabular}

strain NAL8 underlined; bootstrap values of the main internodes (500 replicates) are shown; cluster denominations refer to Rep protein groups of Supplementary Material S4. b ClustalW alignments of iteron sequences from Bifidobacterium plasmids
Fig. 4 Bifidobacterium plasmid iterons. a Neighbor-joining dendrogram generated from the ClustalW alignment of iteron-containing DNA regions from Bifidobacterium plasmids; pNAL8L iterons shown in white letters on black field with two other plasmids of B. longum 
introduced pGOSBif33 via electroporation into B. longum NCC2705 and B. animalis ScMa7, we obtained transformation rates of 200 and 50 clones per microgram of recombinant DNA, respectively. The clones showed bifidobacterial morphology under the microscope, registered positive in the PCR for the erythromycin resistance gene, ery ${ }^{r}$; and we could isolate the expected circular DNA molecule from their cultures (Supplementary Material S6). Moreover, we could accurately identify the plasmid molecules in recombinant cells in PCR experiments with several pairs of primers targeting different regions of pGOSBif33 (data not shown).

\section{Discussion}

Plasmid multiple colonization is quite common in B. longum so that Sgorbati et al. (1982) reported strains exhibiting as many as seven plasmid bands. Nevertheless, no more than two co-resident plasmids of a single strain have been fully characterized so far (Corneau et al. 2004; Lee and O'Sullivan 2006; Park et al 1997). That is why we decided to perform a molecular characterization of the three plasmids harbored by B. longum NAL8, a strain isolated as a member of the predominant bifidobacterial population in a single fecal sample from a healthy elderly woman (Canzi et al. 2005).

Plasmids pNAL8H and pNAL8M showed a close sequence similarity to and the same gene organization as the already characterized $B$. longum plasmids. Plasmids sharing close sequence similarity have already been isolated from $B$. longum strains of different origin (for example, plasmids pB44, pKJ36, pNAC2, pTB6, and pMG1), indicating that molecular recombination and rearrangements occur rarely. Yet, examples of evident rearrangements in B. longum plasmids have been detected by analyzing the pNAL8L DNA sequence or the recently characterized pDOJH10L plasmid (Lee and O'Sullivan 2006).

The phylogenetic analysis of replication proteins supports the hypothesis that ancestral replicons from different hosts may be acquired by and then developed in the Bifidobacterium, resulting in the five Rep types identified (Supplementary Material S4 and Fig. 3). It is also noteworthy that Corynebacterium replicons, known to replicate in bifidobacteria (Argnani et al. 1996; Schürch 2002), contain replication proteins similar to typologies II, III, and IV (Fig. 3), suggesting that these replicons may have begun replicating in members of Bifidobacterium and Corynebacterium before the two evolved into distinct genera. However, these speculations lack experimental proof because the direction of acquisition cannot be inferred from comparative analysis.

Shuttle vectors based on the ColE1 replication origin constitute the most commonly used system to isolate functional replicons of Bifidobacterium (Matteuzzi et al.
1990; Rossi et al. 1996; Matsumura et al. 1997; Park et al. 1997, 1999; Tanaka et al. 2005). However, when ColE1 was used successfully, it was obtained from pBR322, not from high-copy-number pUC vectors. In fact, the experimental data by Corneau et al. (2004) clearly indicate that ColE1 from the high-copy-number vector pUC18 can be rescued from Bifidobacterium. Further, the authors suggested that various replicon-screening systems should be developed to overcome such potential incompatibility problems. Despite several efforts in our laboratory to create E. coli-Bifidobacterium shuttle vectors based on the ColE1 replication origin from pUC vectors, we were equally unable to obtain stable Bifidobacterium transformants. The recombinants on selective agar plates showed bifidobacterial morphology under microscopic observation, were resistant to the antibiotic used for selection, and registered positive in PCR for the antibiotic resistance gene, yet, after plasmid DNA extraction, yielded no expected recombinant DNA molecules (data not shown).

We, thus, prepared a circular DNA molecule, pGHori, that did not code for any replication protein, yet could replicate in the E. coli strain VE7108. In fact, E. coli VE7108 has on the chromosome a constitutively expressed copy of the replication gene repA. The product of repA acting in trans recognizes the origin still present in pGHori and promotes its replication through a rolling circle mechanism. Based on the E. coli VE7108/pGHori system, we built the pGOSBif33 shuttle vector that was successfully employed for the transformation of B. longum $\mathrm{NCC} 2705$ and B. animalis ScMa7. In conclusion, pGHori and its host, E. coli VE7108, can serve as a system suitable for avoiding possible interference by other functional replication modules on the vector, such as the pUC origin, and consequently, for screening functional replicons from wild-type plasmids.

Acknowledgment We wish to thank Prof. Daniele Daffonchio for the discussions we had with him and for his helpful suggestions, and Dr. Mauro Scarpellini for the technical support. We are also grateful to Drs. Pauli Kallio, Jarmo Niemi, and Mikko Metsä-Ketelä for their kind help at Turku University. Special thanks are due to Prof. Emanuelle Maguin for providing the $\mathrm{pG}^{+}$host vector and E. coli VE7108 and to Nestlé Research Center for providing the strain B. longum NCC2705.

\section{References}

Argnani A, Leer RJ, van Luijk N, Pouwels PH (1996) A convenient and reproducible method to genetically transform bacteria of the genus Bifidobacterium. Microbiology 142:109-114

Canzi E, Guglielmetti S, Mora D, Tamagnini I, Parini, C (2005) Conditions affecting cell surface properties of human intestinal bifidobacteria. Antonie Van Leeuwenhoek 88:207-219

Corneau N, Emond E, LaPointe G (2004) Molecular characterization of three plasmids from Bifidobacterium longum. Plasmid 51:87-100

del Solar G, Giraldo R, Ruiz-Echevarria MJ, Espinosa M, az-Orejas R (1998) Replication and control of circular bacterial plasmids. Microbiol Mol Biol Rev 62:434-464 
Garcý'a de Viedma D, Giraldo R, Rivas G, Fernandez-Tresguerres E, Diaz-Orejas R (1996) A leucine zipper motif determines different functions in a DNA replication protein. EMBO J 15:925-934

Hintermann G, Fischer HM, Crameri R, Hutter R (1981) Simple procedure for distinguishing $\mathrm{CCC}$, OC and $\mathrm{L}$ forms of plasmid DNA by agarose gel electrophoresis. Plasmid 5:371-373

Hofreuter D, Haas R (2002) Characterization of two cryptic Helicobacter pylori plasmids: a putative source for horizontal gene transfer and gene shuffling. J Bacteriol 184:2755-2766

Ingmer H, Fong EL, Cohen SN (1995) Monomer-dimer equilibrium of the pSC101 RepA protein. J Mol Biol 250:309-314

Iwata M, Morishita T (1989) The presence of plasmids in Bifidobacteriumbreve. Lett Appl Microbiol 9:165-168

Lee JH, O'Sullivan DJ (2006) Sequence analysis of two cryptic plasmids from Bifidobacterium longum DJO10A and construction of a shuttle cloning vector. Appl Environ Microbiol 72:527-535

Maguin E, Prévost H, Ehrlich SD, Gruss A (1996) Efficient insertional mutagenesis in lactococci and other Gram-positive bacteria. J Bacteriol 178:931-935

Matsumura H, Takeuchi A, Kano Y (1997) Construction of Escherichia coli-Bifidobacterium longum shuttle vector transforming B. longum 105-A and 108-A. Biosci Biotechnol Biochem 61:1211-1212

Matteuzzi D, Brigidi P, Rossi M, Di Gioia D (1990) Characterization and molecular cloning of Bifidobacterium longum cryptic plasmid pMB1. Lett Appl Microbiol 11:220-223
Park MS, Lee KH, Ji GE (1997) Isolation and characterization of two plasmids from Bifidobacterium longum. Lett Appl Microbiol 25:5-7

Park MS, Shin DW, Lee KH, Ji GE (1999) Sequence analysis of plasmid pKJ50 from Bifidobacterium longum. Microbiology 145:585-592

Rossi M, Bridigi P, Gonzalez Vara y Rodriguez A, Matteuzzi D (1996) Characterization of the plasmid pMB1 from Bifidobacterium longum and its use for shuttle vector construction. Res Microbiol 147:133-143

Sambrook J, Russell DW (2001) Molecular cloning, a laboratory manual. Cold Spring Harbor Laboratory, Cold Spring Harbor

Schürch C (2002) Development of a novel DNA transformation system for bifidobacteria. A dissertation submitted to the Swiss Federal Institute of Technology Zurich Ethz for the degree of Doctor of Technical Sciences. Prof. Dr. Michael Teuber, examiner; PhD Dr. Hans-Martin Fischer, co-examiner; PhD Dr. Leo Meile, co-examiner, Zurich. http://e-collection.ethbib.ethz. ch/cgi-bin/show.pl?type $=$ diss\&nr $=14676$

Sgorbati B, Scardovi V, Leblanc DJ (1982) Plasmids in the genus Bifidobacterium. J Gen Microbiol 128:2121-2131

Stanton C, Gardiner G, Meehan H, Collins K, Fitzgerald G, Lynch PB, Ross RP (2001) Market potential for probiotics. Am J Clin Nutr 73:476S-483S

Tanaka K, Samura K, Kano Y (2005) Structural and functional analysis of pTB6 from Bifidobacterium longum. Biosci Biotechnol Biochem 69:422-4253 